\title{
Open and Association MCTAs Access and Allocation Scheme by Staggering Algorithm in IEEE 802.15.3
}

\author{
Eui-Seok Hwang ${ }^{1}$, You-Chang $\mathrm{Ko}^{2}$, Choong-Ho $\mathrm{Cho}^{3}$, \\ Hyong-Woo Lee ${ }^{4}$, and Sumit Roy ${ }^{1}$ \\ ${ }^{1}$ Univ. of Washington Dept. of Electrical Engineering \\ \{eui, roy\}@ee.washington.edu \\ ${ }^{2}$ LG Electronics Inc. Mobile Handset R\&D Center \\ ycko@lge.com \\ ${ }^{3}$ Korea Univ. Dept. of Computer \& Information Science \\ chcho@korea.ac.kr \\ ${ }^{4}$ Korea Univ. Dept. of Electronics \& Information Engineering \\ hwlee@korea.ac.kr
}

\begin{abstract}
The IEEE 802.15.3 medium access control (MAC) protocol is standard for high bit rate wireless personal area network (WPAN). The open or association management channel time allocations (MCTAs) are used by devices(DEVs) for sending command messages or association request command to piconet coordinator (PNC) by means of slotted aloha random access manner. Based on slotted aloha scheme the binary back-off algorithm has been considered as a primary contention resolution candidate due to its simple operation. However it is not appropriate for the future wireless networks because of low throughput and high delay and delay variance. Without loss of generality the goals of the random multiple access algorithm are to maximize the throughput and to minimize the average packet delay. In this paper, we propose a new multiple access protocol named staggering algorithm working on top of slotted aloha scheme. The performance results by NS-2 simulation show that the proposed algorithm achieves the maximum throughput up to 0.54 and guarantees the QoS in terms of delay and delay variance of realtime multimedia traffic.
\end{abstract}

\section{Introduction}

IEEE 802.15.3 working group [1] is working on technologies targeted at enabling high bit rate multimedia applications operating in WPAN. These technologies include both MAC and PHY protocols that enable WPAN to support up to 243 DEVs operating at least $20 \mathrm{Mb} / \mathrm{s}$ [2]. IEEE 802.15 .3 piconet is a wireless ad hoc data communications system which allows a number of independent data DEVs to communicate with each other. To provide multimedia QoS, a TDMA-based superframe structure is adopted. The superframe is composed of three major parts: the beacon, the optional contention access period (CAP) and the channel 
time allocation period (CTAP), as shown in Figure 1. Any DEV associated in the piconet may attempt to send a command frame to the PNC in an open MCTA. Any DEV not currently associated in the piconet also may attempt to send an association request command to the PNC in an association MCTA. It is the PNC's responsibility to determine the number of MCTAs to use for each superframe. It is desirable that the number of MCTAs is dynamically adapted by the PNC depending on the current traffic conditions. As a random access scheme slotted aloha was proposed for an open MCTA or an association MCTA in [2]. However, this mechanism in line with the exponential back-off algorithm for a collision resolution has been challenged by some prior works in order to enhance realtime multimedia traffic access efficiently in terms of throughput, delay, and delay variance[4-6]. In this paper, we propose a new open and association MCTAs access and allocation scheme named staggering algorithm to deal with collision resolution which is inevitable in random access environment such as slotted aloha scheme. NS-2 simulation results show that the proposed scheme provides lower mean delay/delay variation and higher throughput than previous schemes. The throughput of the proposed scheme is remarkably increased up to 0.54 .

This paper is organized as follows: Section 2 provides slotted aloha access scheme for open and association MCTAs followed by a description of previous proposed schemes. Section 3 describes the proposed scheme. We present simulation results in section 4 . Section 5 concludes the paper.

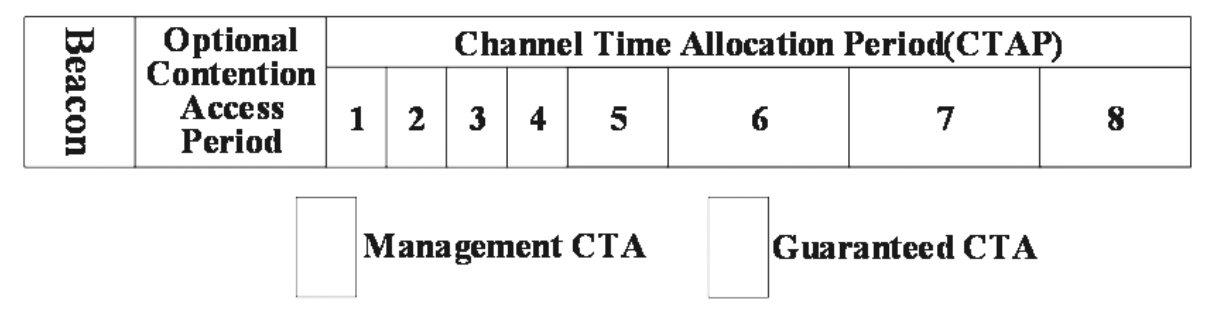

Fig. 1. Superframe structure

\section{Related Works}

\subsection{Slotted Aloha Access for Open and Association MCTAs [2]}

The access to an open or association MCTA shall be controlled by a contention window $C W_{a}$ maintained by each DEV. Each DEV decides $C W_{a}$ by the number $a$, where $a$ is the number of retransmission attempts made by the DEV. For the first access attempt, $a$ shall be set to zero. The size of the contention window, $C W_{a}$ is defined as follows,

$$
C W_{a}= \begin{cases}256 & 2^{a+1} \geq 256 \\ 2^{a+1} & 2^{a+1}<256\end{cases}
$$


The open or association MCTA used for the $a^{\text {th }}$ retransmission attempt shall be chosen by a uniformly distributed random integer value $r$ within the interval $\left[1, C W_{a}\right]$. The DEV shall start counting $r$ beginning with the open or association MCTA in the current superframe and continue across superframes. The open or association MCTA with number equal to $r$ is the MCTA that the DEV shall access. The DEV shall not access the MCTA before its counter has reached the open or association MCTA with the number equal to $r$. After receiving the ACK, $a$ will be reset to 0 . This retransmission scheme based on slotted aloha is simple but inappropriate for the future wireless networks because of low throughput and high delay variance.

\subsection{Previous Scheme [3]}

Recently, extensive research has been devoted to IEEE 802.15.3 [6-10]. In this paper we introduce a new random access scheme in IEEE 802.15.3. The random access scheme being used by HIPERLAN/2 is much similar to that of IEEE 802.15.3 such a way that both of them use the centralized random access method controlled by PNC or access point (AP) base on slotted aloha scheme. So, we simply compare one of recently developed random access schemes in HIPERLAN/2 with the proposed scheme.

In [3], an AP controls the number of random channels (RCHs), which works as open or association MCTA in IEEE 802.15.3 does, based on the binary splitting algorithm. By the binary splitting algorithm the number of RCHs of $(t+1)^{t h}$ MAC frame is given by

$$
r(t+1)=\min \left\{N_{a}+2 \times N_{f}(t), R_{\mathrm{MAx}}\right\}
$$

where

- $r(t)$ : the number of allotted RCHs at MAC frame $t$

- $N_{f}(t)$ : the number of collided RCHs at MAC frame $t$

- $N_{a}$ : the fixed number of RCHs allocated for newly arriving packets

- $R_{M A X}$ : the maximum number of RCHs per MAC frame

For each collided RCH in the previous frame, additional two RCHs are allocated for the collision resolution. For initial attempt, a mobile terminal (MT), which corresponds to a DEV in IEEE 802.15.3, randomly accesses one RCH within the interval $\left[1, N_{a}\right]$ as Eq. 3 . The collided MTs in the previous MAC frame may choose the RCH to access based on the location information of contention slots where collisions occur. That is, the MTs in the $i^{t h} \mathrm{RCH}$ among the collided RCHs in the previous MAC frame would randomly access either $\left(2 \times i-1+N_{a}\right)^{t h}$ or $\left(2 \times i+N_{a}\right)^{t h} \mathrm{RCH}$ as Eq. 4 . In addition, if there are not enough RCHs in the current MAC frame, the retransmission occurs within the interval $\left[1, N_{a}\right]$ after a random delay by frame unit as Eq. 5 .

$\diamond$ Initial attempt: Random access within $\left[1, N_{a}\right]$

$\diamond$ Retransmission: 
Random access either $2 i-1+N_{a}$ or $2 i+N_{a} \quad$ if $2 i+N_{a} \leq R_{\mathrm{MAX}}$

Random access within $\left[1, N_{a}\right]$ after a random delay if $2 i+N_{a}>R_{\mathrm{MAX}}$

where $i$ is the location of collided $\mathrm{RCH}$ in the previous MAC frame.

\section{Proposed Scheme: Staggering Algorithm}

In the proposed scheme the structure of time slot is changed such a way that each slot is separated into two areas, the front part and the rear one, and most of their parts are overlapped each other. The collided DEVs by accessing the front part of a slot in the previous superframe would access any part of the first slot of additional two slots prepared by binary splitting for the collision resolution. The collided DEVs by accessing the rear part would access any part of the second slot of two additional slots. If all collided DEVs are in the same part of slot, they choose any part of any two additional slots randomly.

The PNC controls the number of MCTAs based on the splitting algorithm. The number of MCTAs of $(t+1)^{t h}$ superframe is given by

$$
r(t+1)=\min \left\{N_{a}+2 \times N_{f}(t), R_{\mathrm{MAX}}\right\}
$$

where

- $r(t)$ : the number of allotted MCTAs at superframe $t$

- $N_{f}(t)$ : the number of collided MCTAs at superframe $t$

- $N_{a}$ : the fixed number of MCTAs allocated for newly arriving packets

- $R_{M A X}$ : the maximum number of MCTAs per superframe

There are $N_{a}$ MCTAs for newly arriving request packets in each superframe. For each collided MCTA in the previous superframe, additional two MCTAs are allocated for the collision resolution. For the initial attempt, a DEV randomly accesses any part of one MCTA within the interval $\left[1, N_{a}\right]$ as Eq. 7. If a collision occurs, the collided DEVs in the previous superframe choose the MCTA to access again based on the location information of contention slots where collisions occur. That is, if all collided DEVs are in the same part of the $i^{\text {th }}$ MCTA among the collided MCTAs in the previous superframe, they randomly access any part of either $\left(2 \times i-1+N_{a}\right)^{t h}$ or $\left(2 \times i+N_{a}\right)^{t h}$ MCTA as Eq. 9. Otherwise the collisions are occurred in both parts of slot, the collided DEVs in the front part of slot access any part of $\left(2 \times i-1+N_{a}\right)^{t h}$ MCTA as Eq. 10. and the others access any part of $\left(2 \times i+N_{a}\right)^{t h}$ MCTA as Eq. 11. In addition, if there are not enough MCTAs in the current superframe, the retransmission occurs within the interval $\left[1, N_{a}\right]$ after a random delay by frame unit as Eq. 8 .

$\diamond$ Initial attempt: Random access within $\left[1, N_{a}\right]$

$\diamond$ Retransmission:

- If $2 \times i+N_{a}>R_{\mathrm{MAX}}$ access any part of slot within $\left[1, N_{a}\right]$ after a random delay, where $i$ is the location of the collided MCTA in the previous superframe. 
- Else if all collided DEVs are in the same part access any part of either $i \times 2-1+N_{a}$ or $i \times 2+N_{a}$

- Else if collided DEVs are in the front of slot access any part of $i \times 2-1+N_{a}$

- Else if collided DEVs are in the rear of slot access any part of $i \times 2+N_{a}$

Fig. 2 shows an example of how the number of MCTAs would be decided by the staggering algorithm, in which the number of MCTAs for new requests is assumed by two and $R_{M A X}$ is eight. If four MCTAs are collided in the previous superframe, the number of MCTAs will be 10 in the current superframe(two MCTAs for new requests and eight MCTAs that are splitted by four collided MCTAs). However, the number of MCTAs in the current superframe should be eight because $R_{M A X}$ is limited by eight.

In the current superframe, new DEVs can access the first or second MCTA. The DEVs collided on the front part of the first slot, $C_{11}$ of the previous superframe access any part of the $3^{r d}$ slot. The DEVs collided on the rear part of the first slot, $C_{12}$ of the previous superframe access any part of the $4^{\text {th }}$ slot. Similarly, the DEVs collided on $C_{21}$ of the previous superframe access any part of the $5^{\text {th }}$ slot. The DEVs collided on $C_{22}$ of the previous superframe access any part of the $6^{\text {th }}$ slot. Since all collisions are occurred in the front part of the $4^{\text {th }}$ slot of the previous superframe, the DEVs of $C_{31}$ randomly access either the $7^{\text {th }}$ or the $8^{\text {th }}$ slot. However, the DEVs involved in $C_{42}$ in the previous superframe cannot access any MCTA slot in the current superframe because the maximum number of MCTAs is limited by eight. In this case, these DEVs should access MCTAs in $N_{a}$ area as if they are newly arrived packets after a random delay. After all, the proposed scheme can resolve collisions quickly by means of reducing randomness by staggering algorithm when DEVs access MCTAs.

\section{Simulation Results}

This section presents some NS-2 simulation results on the throughput, average delay, and delay variance. For simulation, we assume that there is no transmission error due to radio channel environment, there are one PNC and $50 \mathrm{DEVs}$ as a whole, and each DEV generates the message according to Poisson process with rate $\lambda$ per superframe. We also assume a DEV cannot generate a new request message until the access attempt succeeds. Throughout this section the (normalized) throughput, $\rho$, is defined as the fraction of MCTAs in which successful transmissions occur.

In Figs. 3, we observe that the maximum throughput of the proposed scheme is $54 \%$, while that of [3] is $47 \%$ and fixed MCTA allocation scheme is $36 \%$. Slotted aloha access mechanism [2] is adapted for fixed MCTA allocation scheme in Fig. 3. Therefore, the proposed scheme utilizes the MCTAs more efficiently than previously proposed schemes. The number of allocated MCTA is one of $[1,2$, $4,8,16,32]$ in the fixed MCTA allocation scheme. The maximum throughput can be got when the number of MCTA is small if the offered load is low and 




Fig. 2. An example of the staggering algorithm $\left(N_{a}=2 R_{M A X}=8\right)$

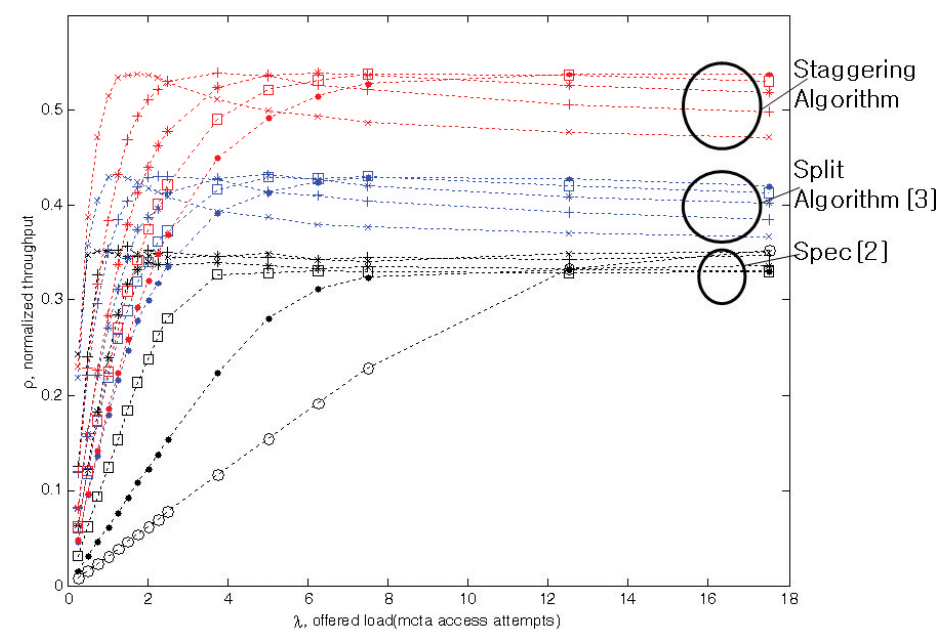

Fig. 3. Offered load versus throughput

(Staggering Alog. VS. Split Algo. VS. Fixed MCTA allocation scheme)

vice verse. However, it can be anticipated easily the maximum throughput of these networks is at most 1/e and high delay and standard deviation of delay because the basic MAC protocols of these networks are slotted ALOHA with back-off. Several lines of staggering and split algorithm result from different $N_{a}$. In general, when the MCTA access attempt is low, the higher throughput can be achieved when $N_{a}$ is low. That is, allocating many MCTAs for new attempts is a 


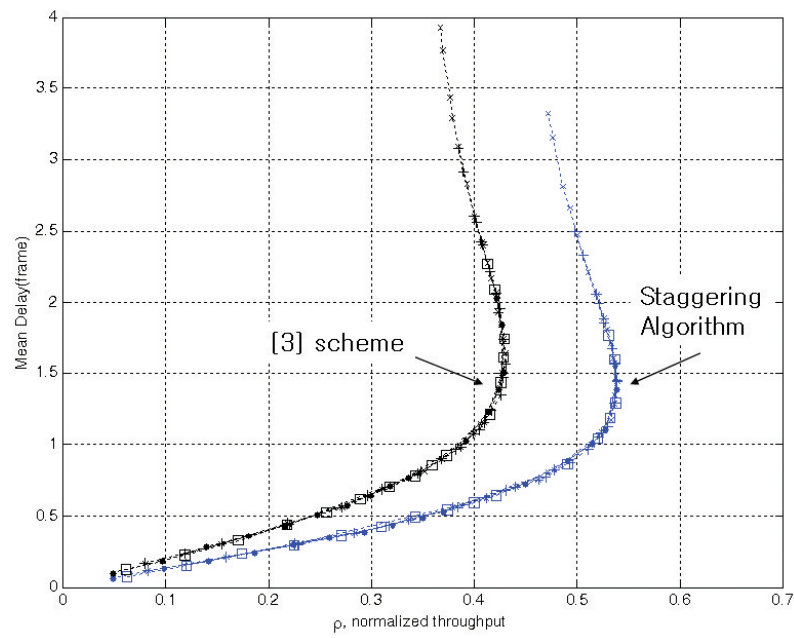

Fig. 4. Throughput versus mean delay (Staggering Algo. VS.[3])

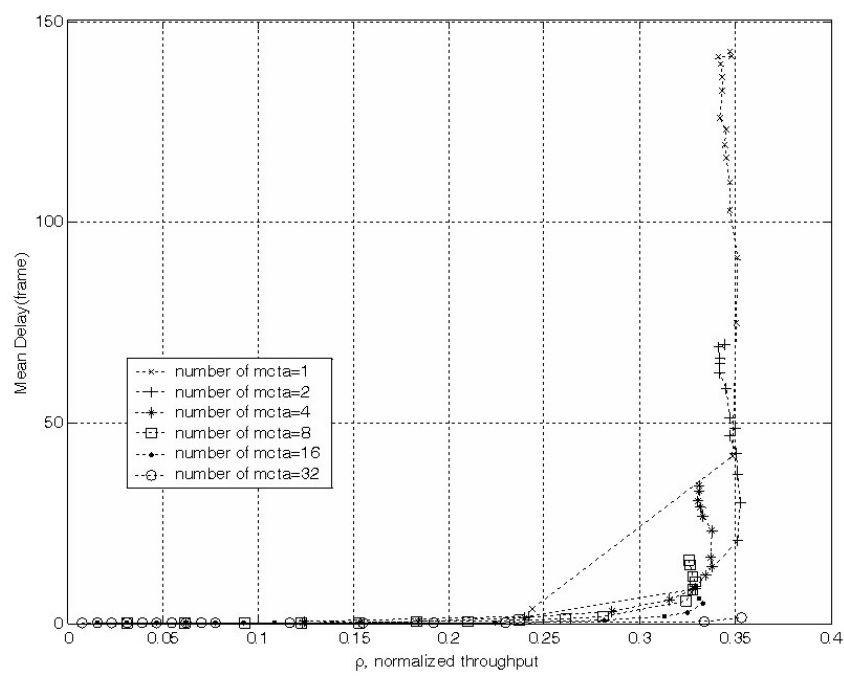

Fig. 5. Throughput versus mean delay (Fixed MCTA allocation scheme)

waste of resources when access attempt is low. When the MCTA access attempt is high, throughput can be improved when $N_{a}$ is high.

Fig. 4 shows the throughput-delay characteristics of the proposed scheme. For example, the proposed scheme shows the delay within one frame when throughput is 0.4 , however, the previous scheme [3] shows the delay beyond one frames. Of course, extreme delay is shown in the fixed MCTA allocation scheme when the number of allocated MCTA is small as shown in Fig. 5. Real-time traffic like streaming video and audio as well as best-effort traffic is anticipated to occupy 


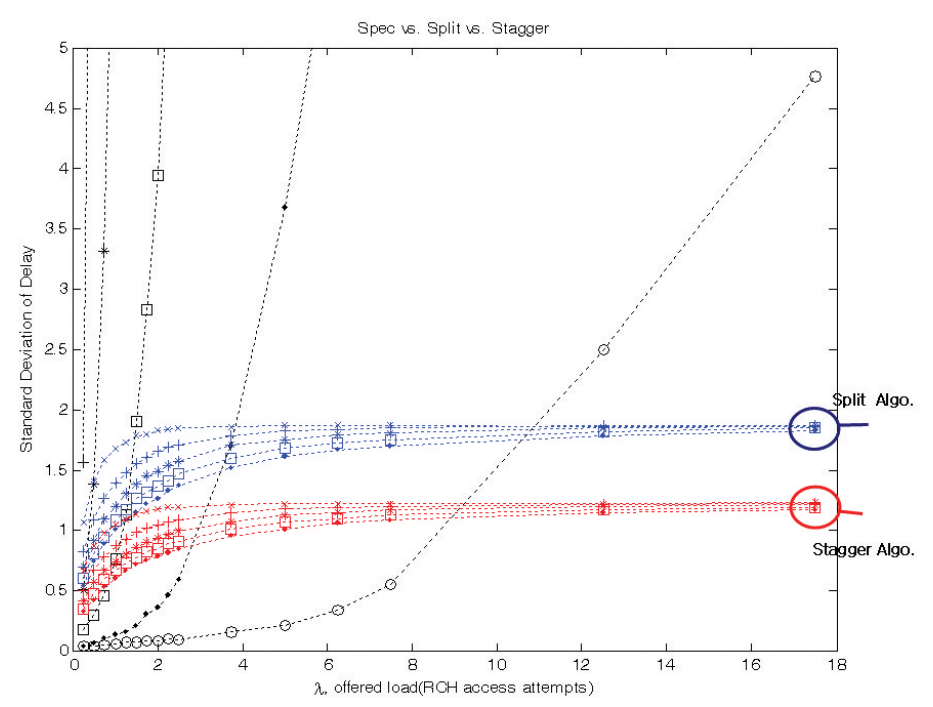

Fig. 6. Offered load versus delay variance

a large portion of WPAN traffic[11]. Therefore, we can expect that the proposed scheme can be useful in guaranteeing QoS of multi-media traffic in WPAN.

Fig. 6 describes the proposed staggering algorithm can guarantee the delay jitter. Standard deviation of delay can be an indicator of sharing the limited resource equally and another importance parameter in the next generation wireless networks. The different lines in the same algorithm are the difference of $N_{a}$. Back-off algorithm allows a single or a few winning user to dominate the available bandwidth. Therefore the shared channel can be used unfairly in the back-off algorithm.

\section{Conclusion}

The slotted aloha scheme with exponential back off algorithm for the IEEE 802.15.3 is simple to implement. Yet, it has the relatively low maximum throughput of $1 / \mathrm{e}$ and suffers from large mean and variance of access delay. For the next generation of wireless networks with multimedia services, the delay throughput performance of the slotted aloha scheme with exponential back off needs to be improved. In this paper, we have proposed a new open and association MCTA access and allocation scheme. Through computer simulations, we have shown that the proposed staggering algorithm achieves the maximum throughput as high as 0.54 . The result, when compared with previously proposed algorithms $[2,3]$ gains more than 0.1 . The proposed scheme also performs well in terms of delay and delay variance. This can be significant for QoS guarantee for delay sensitive real time multimedia traffic in WPAN. 


\section{References}

1. IEEE, "802.15 WPAN task group 3(TG3)", http://www.ieee802.org/15/pub/ TG3.html

2. IEEE 802.15.3 Working Group, "Part 15.3: Wireless Medium Access Control (MAC) and Physical Layer (PHY) Specifications for High Rate Wireless Personal Area Networks (WPAN)." IEEE Computer Society, Sep. 2003

3. Eui-Seok Hwang, et al: "Random Channel Allocation Scheme in HiperLAN/2", LNCS $33312532 \mathrm{pp}, 2004$

4. You-Chang Ko et al: "Collision Reduction Random Access Using m-ary Split Algorithm in Wireless Access Network" LNCS3510, pp.223 233, 2005.

5. Gyung-Ho Hwang, Dong-Ho Cho: "Adaptive Random Channel Allocation Scheme in HIPERLAN/2 ", IEEE COMMUNICATIONS LETTERS, VOL. 6, NO. 1, JAN. 2002, pp. $40-42$

6. Kwan-Wu Chin and D. Lowe: "Simulation Study of the IEEE 802.15.3 MAC", Australian Telecommunications and Network Applications Conference (ATNAC), Sydney, Australia, December, 2004

7. Seung H. Rhee, K. Chung, Y. Kim, W. Yoon, and K. S. Chang: "An ApplicationAware MAC Scheme for IEEE 802.15.3 High-Rate WPAN", IEEE WCNC, Mar. 2004

8. Byung S. Kim, Y. Fang and Tan F. Wong: "Rate-Adaptive MAC Protocol in HighRate Personal Area Networks" , IEEE WCNC, Mar. 2004

9. Attila Torok, Lorant Vajda, Kyu J. Youn and Sun-Do June: "Superframe Formation Algorithms in 802.15.3 Networks", IEEE WCNC, Mar. 2004

10. Xin Wang, Yong Ren, Jun Zhao, Zihua Guo and Richard Yao: "Comparison of IEEE 802.16e and IEEE 802.15.3 MAC", IEEE 6th CAS Symp. On Emerging Technologies, Shanghai, China, May, 2004

11. P. Gandolfo and J. Allen: "802.15.3 Overview/Update," The WiMedia Alliance, Oct. 2002 